Research Article

\title{
Residual Life Prediction of Metro Traction Motor Bearing Based on Convolutional Neural Network
}

\author{
Yanwei Xu $\mathbb{D}^{1,2}$ Weiwei Cai, ${ }^{1}$ Tancheng Xie, ${ }^{1,2}$ and Pengfei Zhao' \\ ${ }^{1}$ School of Mechatronics Engineering, Henan University of Science and Technology, Luoyang 471003, China \\ ${ }^{2}$ Intelligent Numerical Control Equipment Engineering Laboratory of Henan Province, Luoyang 471003, China \\ Correspondence should be addressed to Yanwei Xu; xuyanweiluoyang@163.com
}

Received 15 May 2021; Revised 1 July 2021; Accepted 19 July 2021; Published 26 July 2021

Academic Editor: Wenxian Yang

Copyright (c) 2021 Yanwei Xu et al. This is an open access article distributed under the Creative Commons Attribution License, which permits unrestricted use, distribution, and reproduction in any medium, provided the original work is properly cited.

In order to solve the problem that a single type of sensor cannot fully reflect the bearing life information in the process of bearing residual life prediction of metro traction motor, a bearing residual life prediction method based on multi-information fusion and convolutional neural network is proposed. Firstly, the vibration sensor and acoustic emission sensor are used to collect the bearing life signals on the bearing fatigue life test bench. Secondly, wavelet packet decomposition is used to denoise the collected bearing life signal and extract multiple eigenvalues. On this basis, the multiple eigenvalues are normalized, and the bearing degradation trend is analyzed. Finally, the collected bearing life is divided into five stages, and the processed multiple eigenvalues are fused and input into convolutional neural network for training and recognition. The results show that the probability of predicting the stage of bearing life based on multiple eigenvalues and convolutional neural network is more than $98 \%$.

\section{Introduction}

Rolling element bearings are one of the most critical components in rotating machinery to support rotating shafts. Any unexpected failure of bearings may result in several negative implications, such as downtime increase, productivity reduction, and even raise of safety risks [1-5].

In recent years, great achievements have been made in the research of algorithms in various fields. For example, Deng et al. [6] proposed an improved MSIQDE algorithm based on hybrid Multistrategy to solve the problem that quantum differential evolution (QDE) is easy to lead to premature convergence and low search ability and fall into local optimum. Aiming at the problems of slow convergence speed, poor global search ability, and difficulty in designing rotation angle of quantum heuristic evolutionary algorithm (QEA), Cai et al. [7] proposed an improved quantum cooperative coevolution algorithm with faster convergence speed and higher convergence accuracy based on coevolution strategy. Deng et al. [8] and others proposed an improved differential evolution algorithm (HMCFQDE) in order to overcome the shortcomings of differential evolution algorithm, such as low efficiency, insufficient search diversity, slow convergence speed, and high possibility of search stagnation, combined with the quantum computing characteristics of quantum evolutionary algorithm (QEA) and the idea of divide and rule of cooperative coevolutionary algorithm (CCEA). Based on the nonlinear Wiener process model, Wen et al. [9] and others proposed an improved method to predict the remaining service life of bearings. Chen [10] and others proposed a prediction model based on correlation features and multivariable support vector machine to solve the problem of using small samples to predict the residual life of rolling bearings due to the lack of sufficient condition monitoring data. Zhao et al. [11] proposed KELM (maximum power spectral density fitting curve differentiation) and weight applied to time to failure (WAFT) to solve the problem of different individual methods of rolling bearings and the different performance degradation curves caused by the difference of kernel extreme learning quality and working conditions and established a new RUL prediction model of rolling bearings. Yang and Court [12] conducted a study on the actual health status of test bearings with different types and severity of failures by 
using four dimensionless status monitoring standards. These studies have achieved good results in the direction of bearing life prediction, but they all input a single eigenvalue into the model for training, the extracted bearing life information is limited, and the multieigenvalue information fusion technology is not considered. The multieigenvalue information fusion technology can improve the prediction accuracy. With the development of information fusion technology, more and more scholars join in the research. Wu and Zhang [13] proposed a new cascaded fusion convolutional longand short-term memory network for orientation rule prediction because of the limited structure of current deep learning methods and the poor stability of prediction results due to the use of single sensory data. Wang et al. [14] used KPCA method to reduce the dimension of the extracted features and used kernel principal component to estimate the parameters of WPHM model and proposed a trend prediction method of rolling bearing residual service life based on Pchip-EEMD-GM(1, 1). These studies using information fusion technology in the direction of bearing residual life prediction have achieved good results and provide useful reference in information fusion technology, but, using a single type of sensor, the information collected cannot fully reflect the bearing life information, which will affect the prediction of bearing life.

In order to solve the problem that a single sensor cannot fully reflect the bearing life information, this paper uses wavelet packet decomposition [15] to denoise the collected original signal and extract multiple eigenvalues and studies the remaining life prediction of metro traction motor bearing based on information fusion and convolutional neural network.

\section{Signal Preprocessing and Degradation Index Selection}

2.1. Signal Acquisition and Preprocessing. The model of the bearing studied in this paper is $\mathrm{Nu} 216$. As the bearing of metro traction motor, it plays an important role in the safe operation of the subway, so it is particularly important to predict the remaining service life of the bearing. In order to reduce the test cycle and simulate the early defects in the actual operation of the bearing, the Da Zu YLP-MDF-152 three-dimensional laser instrument was used to prefabricate the defects on the rolling element. During the process of defect prefabrication, $30 \%$ laser energy was used. The pitting diameter was $20 \mu \mathrm{m}$ and the crack width was $15 \mu \mathrm{m}$. On this basis, the remaining life is explored and the remaining life test is carried out. The selected test bench is T40-120, which is located in Henan Engineering Laboratory of intelligent numerical control equipment. The test bench is composed of hydraulic system (providing loading, cooling, and lubrication), temperature measurement system, pressure measurement system, and data acquisition system. The data acquisition system includes acoustic emission sensor (model: R50S-TC, measurement range: $50 \mathrm{kHz}-700 \mathrm{kHz}$, maximum sampling frequency: $10 \mathrm{~m} / \mathrm{sS}$ ) and vibration sensor (model: LC0151T, range: $33 \mathrm{~g}$, sensitivity: $150 \mathrm{mv} / \mathrm{g}$ ). The test bench is shown in Figure 1.

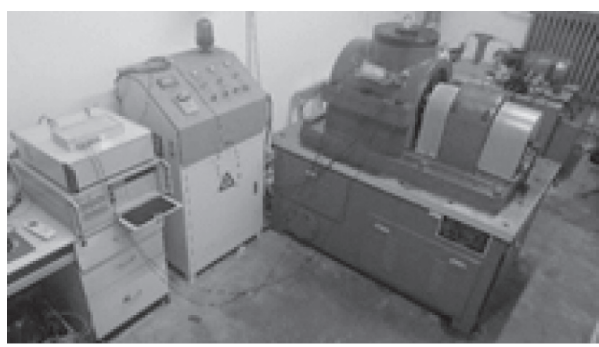

FIGURE 1: Bearing fatigue life test bench.

The test was carried out according to GB/T24607-2009 national test standard. The 120-hour fatigue life strengthening test was carried out under the working conditions of radial equivalent dynamic load of $40 \mathrm{kN}$ and rotating speed of $2400 \mathrm{r} / \mathrm{min}$. The bearing status data is collected every 10 minutes. After the test is completed, a total of 720 sets of bearing performance degradation test data are collected. There are a lot of noises in the collected original signals. In order to filter and denoise the original signals, they are decomposed by wavelet packet. Wavelet packet decomposition is a more detailed time-frequency analysis method. It decomposes the high frequency and low frequency of the original signal at the same time, which ensures that the signal has high resolution in different frequency bands and greatly improves the signal analysis ability [16].

Wavelet packet decomposition is used to decompose the signal $\mathrm{N}$ times, and each decomposition will decompose the high-frequency and low-frequency signals obtained from the previous decomposition. The decomposed signal retains the bearing life information without distortion. Suppose that the eigenvector extracted from a single sensor is $Z_{i}(i=1,2, \ldots, k)$, so the eigenvector obtained by multisensor synthesis is $W \times Z_{i}$. The expansion of $Z_{i}$ is shown in the following formula:

$$
Z_{i}=\left(\begin{array}{ccc}
z_{11} & \ldots & z_{1 k} \\
\vdots & \ddots & \vdots \\
z_{m 1} & \cdots & z_{m k}
\end{array}\right),
$$

where $W$ is the number of sensors, $k$ is the number of extracted eigenvalues, and $m$ is the number of samples. In this paper, db4 wavelet basis function is selected, and the original signal is decomposed by eight times wavelet packet, and the multiple eigenvalues of each frequency band are extracted.

\subsection{Selection of Degradation Index of Bearing Residual Life.} After performing wavelet packet decomposition on the original signal, it is necessary to extract the eigenvalues of the obtained high-frequency and low-frequency signals. In this paper, Fourier transform is used to analyze the original signal in frequency domain, and wavelet packet decomposition is used to process the original signal, so as to extract the characteristic value of time-domain signal. The timedomain features are divided into dimensional and dimensionless. Among them, dimensional time-domain features include standard deviation (SD) and root mean square 
(RMS), and dimensionless time-domain features include kurtosis $(\mathrm{Kr})$ and margin $(\mathrm{C})$. Their formula is as follows:

$$
\begin{aligned}
\mathrm{SD} & =\sqrt{\frac{1}{N} \sum_{i=1}^{N}\left(x_{i}-\frac{1}{N} \sum_{i=1}^{N} x_{i}\right)^{2}}, \\
\mathrm{RMS} & =\sqrt{\frac{1}{N} \sum_{i=1}^{N} x_{i}^{2},} \\
K_{r} & =\frac{1}{N} \sum_{i=1}^{N} x_{i}^{4}, \\
C & =\frac{\sqrt{(1 / N) \sum_{i=1}^{N} x_{i}^{2}}}{(1 / N) \sum_{i=1}^{n} x_{i}} .
\end{aligned}
$$

The standard deviation, root mean square value, kurtosis, and margin are calculated according to the above formula. Some calculation results are shown in Tables 1 and 2 .

From Tables 1 and 2, we can see that the same sensor cannot be directly fused because of the different magnitude of the value between different eigenvalues. At the same time, the numerical unit of the same feature value is different between different sensors, and it cannot be directly fused. Therefore, we need to normalize them to map them to the same interval and perform fusion within the same interval.

\section{Information Fusion and Model Building}

3.1. Fusion of Eigenvalues. In engineering practice, the life decline process of rolling bearings is often reflected by vibration signals, so as to carry out life state identification and life assessment [17]. However, in order to cope with the shortcomings of single type of sensors, vibration and acoustic emission sensors are used to collect bearing life information in this test. After the preprocessed signal and the feature value are extracted, the simultaneous interpreting cannot be directly integrated, because the physical meaning of data collected by different sensors is different, and normalization is needed.

Normalization refers to mapping from one interval to another. Suppose that the original eigenvalue interval extracted is $\left(X_{1}, X_{2}\right)$, and the normalized interval is $\left(Y_{1}, Y_{2}\right)$. If the data before normalization is $G$ and the normalized data is $H$, then the mapping relationship between $G$ and $H$ is as follows:

$$
H=f(G) .
$$

The mapping relation is linear:

$$
H=\frac{\left(G-X_{1}\right)\left(Y_{2}-Y_{1}\right)}{X_{2}-X_{1}}+c .
$$

Information fusion technology is based on normalization and has the characteristics of complementary measurement results of multisensor. Information fusion can
TABLE 1: Characteristic values of bearing life collected by vibration sensor.

\begin{tabular}{lccc}
\hline \multicolumn{4}{c}{ Vibration sensor } \\
SD & RMS & $K_{r}$ & $C$ \\
\hline 43.6529 & 1980.265 & 3.3203 & 1.0473 \\
45.4865 & 1981.92 & 14.5511 & 1.263 \\
52.9402 & 1981.651 & 79.2886 & 1.4892 \\
53.5617 & 1979.754 & 90.9587 & 1.0493 \\
\hline
\end{tabular}

TABLE 2: Characteristic values of bearing life collected by acoustic emission sensor.

\begin{tabular}{lccc}
\hline & \multicolumn{3}{c}{ Acoustic emission sensor } \\
SD & RMS & $K_{r}$ & $C$ \\
\hline 0.0176 & 0.0178 & 687.014 & 170.6823 \\
0.1956 & 0.1961 & 134.212 & 497.5525 \\
0.0454 & 0.0458 & 108.6922 & 111.4096 \\
0.0653 & 0.0653 & 125.7846 & 183.2307 \\
\hline
\end{tabular}

evaluate and decide the importance of its parameters [18]. Information fusion can be divided into data layer fusion, feature layer fusion, and decision level fusion according to the fusion mode. Data layer fusion belongs to the most original fusion [19], and the loss of information is the least. However, due to the difference of detection technology between acoustic emission sensor and vibration sensor, data layer fusion is not adopted; feature level fusion belongs to the fusion of extracted eigenvalues. Although some information is lost, the difference between sensors can be eliminated by normalization processing, and a lot of information will be lost in decision level fusion, so feature level fusion is used in this experiment.

It can be seen from Table 2 that the extracted eigenvalues cannot be directly fused, and they need to be normalized and then fused. After 720 groups of data are fused, 120 groups are selected as the test group, and the remaining 600 groups are taken as the training group. The test group and training group are divided into five stages. The divided test group and training group are marked and input into the neural network for training.

3.2. Prediction Model of Bearing Residual Life Based on MultiInformation Fusion and CNN. Convolutional neural network is a feedforward neural network composed of multilayer networks. In view of the LeNet-5 structure, the convolutional neural network is improved. Initially determine the size of the convolution kernel to be $3 \times 3$; the activation function of convolutional neural network includes sigmoid, $\tan h$, and ReLU, as well as initial selection of activation function ReLU. After the feature information is extracted in convolution layer and transmitted to pooling layer, the pooling layer performs feature selection and information filtering and preliminarily determines that the pooling matrix is $2 \times 2$. The full-connection layer needs to carry out nonlinear combination of the extracted features to get the output, and the output layer selects softmax classification function for classification. The structure of convolutional neural network is shown in Figure 2. 


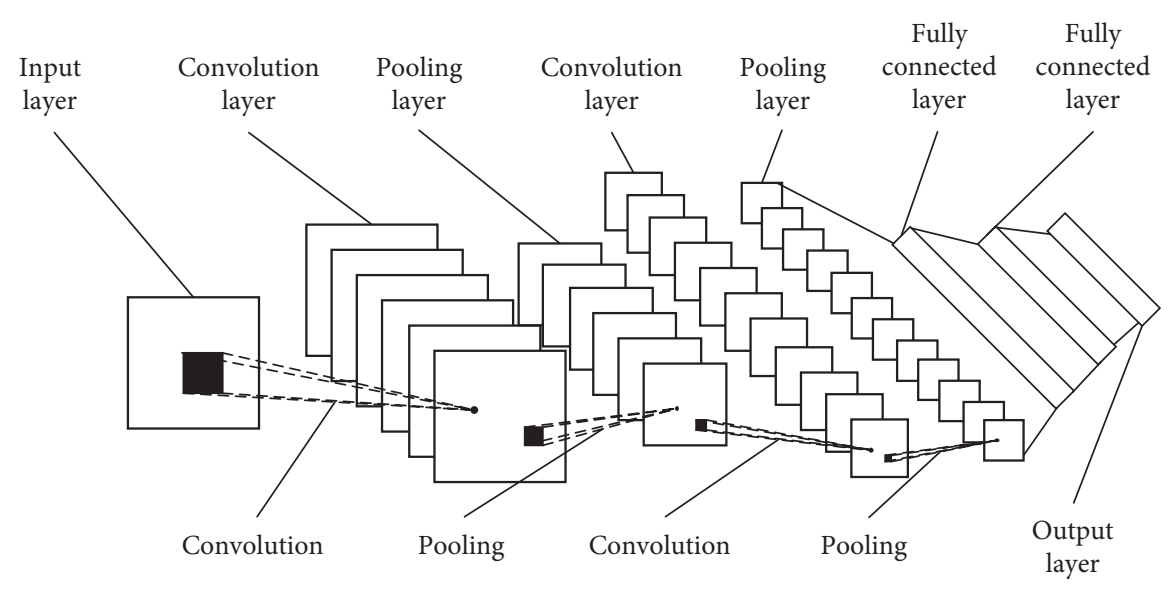

FIgURE 2: Convolutional neural network model.

After normalization, it is necessary to train convolutional neural network, input normalized data into neural network, and adjust the numbers of convolution layers and pooling layers in the convolutional neural network model. Finally, it is determined that the size of the convolution kernel of the first layer of the convolutional neural network is $3 \times 3$, the output dimension is $46 \times 46$, the sliding step is 1 , the pooling matrix of the first layer is $2 \times 2$, and the moving step is 2 . The size of the second layer of convolution kernel is $3 \times 3$, the output dimension is $21 \times 21$, the sliding step is 1 , the pooling matrix of the second layer is $2 \times 2$, the moving step is 2 , and the first layer is fully connected set to 100 , the fully connected layer of the second layer is set to 64, and the output layer is set to 5 . The activation function of the first layer is ReLU, and the activation function of the second layer is $\tan h$. The optimal training times (Epochs) of the model are 100.

After the above work is completed, the normalized eigenvalues of each sensor are input into the adjusted neural network, and the recognition rate is shown in Table 3.

According to Table 3, the recognition rate of different eigenvalues is different. The root mean square value has the best recognition rate, which indicates that the root mean square can extract the most bearing life information, and standard deviation, kurtosis, and margin decrease in turn, which indicates that the bearing life information extracted by them decreases in turn. It can be seen from Table 3 that, for a single sensor, the fusion of different eigenvalues can improve the accuracy of remaining life prediction, which shows that there are similarities and differences between different eigenvalues in extracting bearing life information. Through fusion of feature values extracted by different sensors, it is seen that the accuracy of predicting the remaining life of bearing is also improved, and it is shown that different sensors can be complemented to improve the accuracy of bearing remaining life prediction.

\section{Data Analysis}

By analyzing the normalized eigenvalues, the bearing life trend is shown in Figures 3 and 4.
It can be seen from Figure 3 that, except for kurtosis, the trend of other indicators is upward, which indicates that kurtosis index can extract the least bearing life information, while the root mean square and standard deviation can extract more bearing life information than margin. Figure 4 shows the trend of bearing life collected by AE sensor, and the above conclusion can be verified from Figure 4 .

It can be seen from Figures 3 and 4 that, with the increase of bearing running time, the bearing life index is getting larger and larger. Through the analysis, it can be concluded that dimensionless kurtosis and margin are not as good as dimensionless root mean square and standard deviation. From the two kinds of sensors, vibration sensor is better than acoustic emission sensor in root mean square and standard deviation, and acoustic emission sensor is better than vibration sensor in margin and kurtosis.

It can be seen from Figures 3 and 4 that they have the same bearing life index, and the upper and lower wave momentum are different. In order to explore the influence of the up and down wave momentum on the accuracy, taking root mean square as an example, the root mean square (RMS) obtained from the two sensors is input into the convolutional neural network to obtain the color scale diagram of the accuracy in each stage, as shown in Figure 5.

In the above figure, $A, B, C, D$, and $E$ represent the five stages of bearing life: $A$ represents the first stage, $B$ represents the second stage, $C$ represents the third stage, $D$ represents the fourth stage, and $E$ represents the fifth stage. This experiment is carried out after the discovery of bearing early defects. The first stage is the bearing early defects, and the fifth stage is the final stage of bearing life collection. A total of 720 groups of data were collected, and the collected data were divided into test set and training set, including 120 groups of test samples and 600 groups of training samples. In the training process, the neural network will output the probability values of these five stages for each group of test samples. The 120 groups of test samples will output $120 \times 5$ probability values. Taking an average value of each two groups of 120 groups as the new probability value, the new probability value will be made into a color scale diagram of Figure 5. In this grayscale image, the deeper the color is, the higher the probability is. 
TABLE 3: Recognition rate of each normalized eigenvalue.

\begin{tabular}{lcc}
\hline Eigenvalue & Acoustic emission sensor (\%) & Vibration sensor (\%) \\
\hline RMS & 94.17 & 95.83 \\
$K_{\mathrm{r}}$ & 70.83 & 66.67 \\
C & 70.00 & 61.67 \\
SD & 93.33 & 95.00 \\
Recognition rate of multiple eigenvalues of a single sensor & 95.83 & 96.67 \\
Comprehensive recognition rate of two sensors & & 98.33 \\
\hline
\end{tabular}

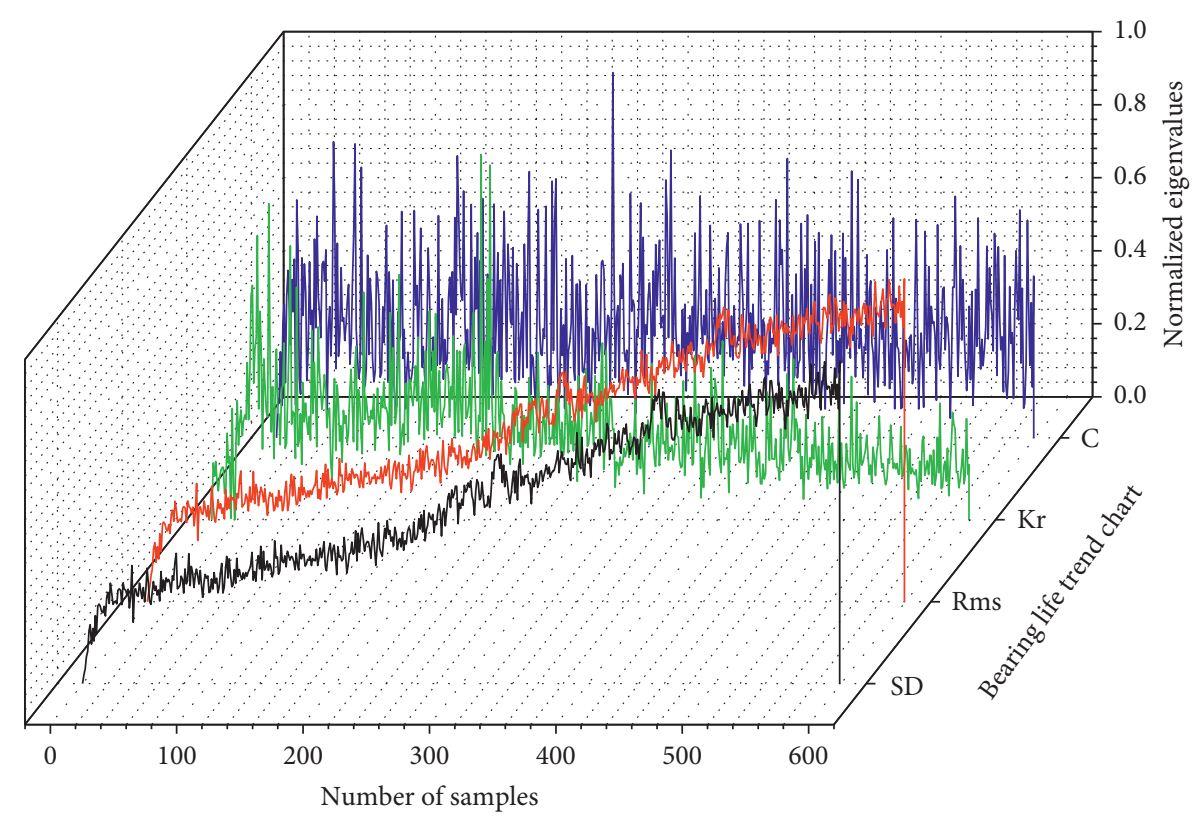

Figure 3: Bearing life curve collected by vibration sensor.

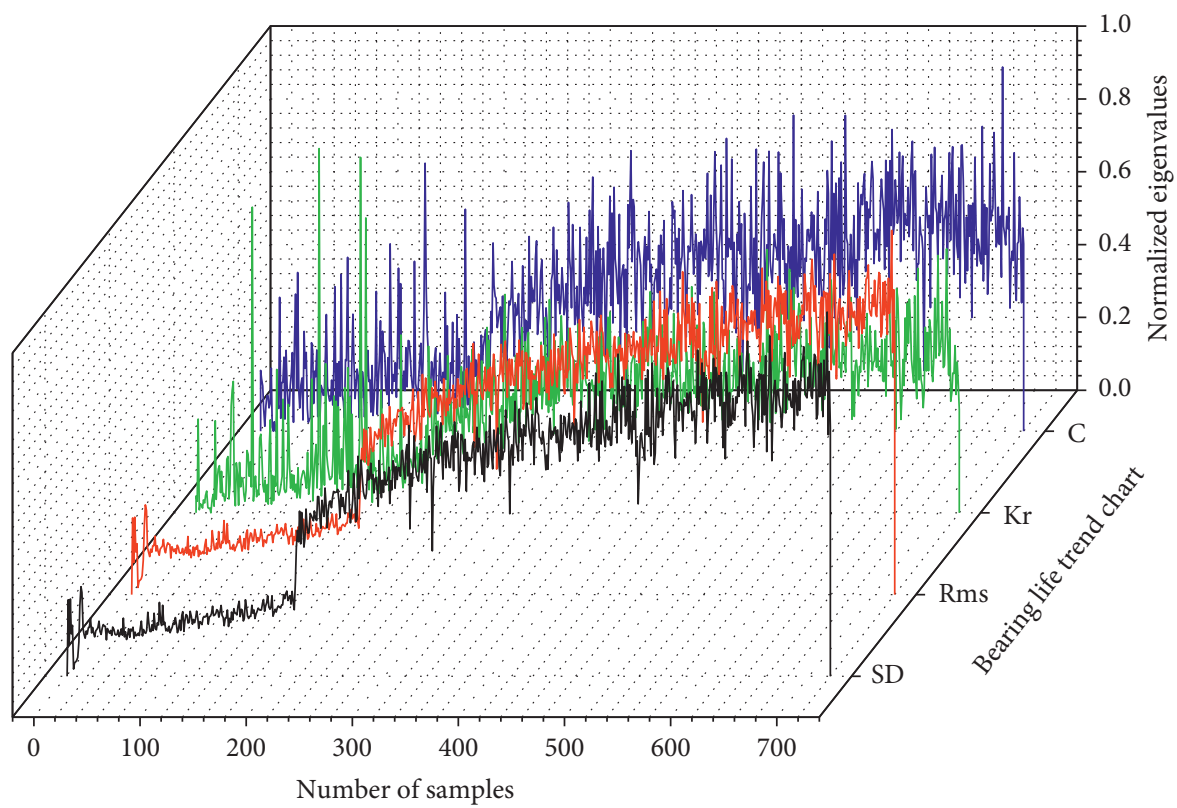

Figure 4: Bearing life curve collected by acoustic emission sensor.

From Figure 5, we can see that the vibration sensor is not as good as the acoustic emission sensor in the first stage and the second stage, but it is better than the acoustic emission sensor in other stages, which shows that the fusion of the information obtained by the two sensors is conducive to improving the accuracy of bearing residual life prediction. 


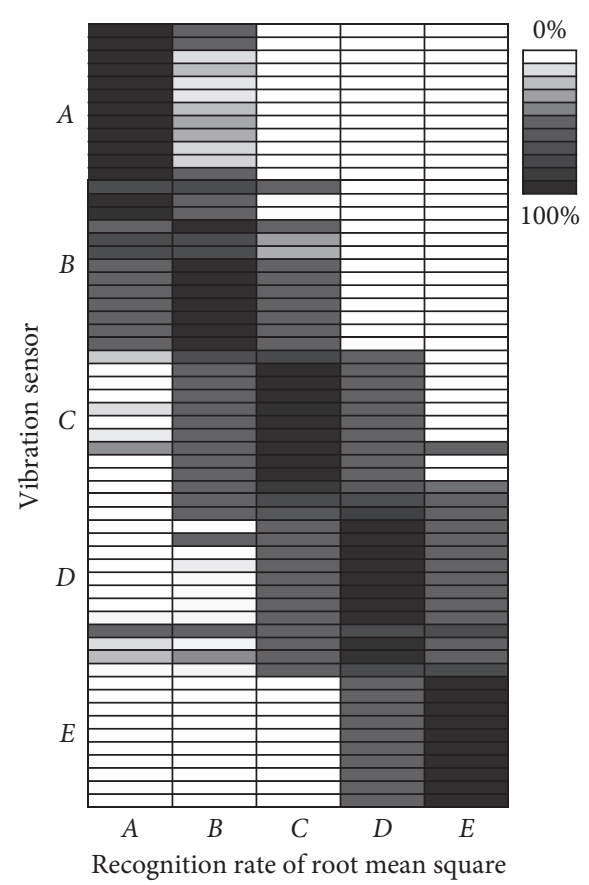

(a)

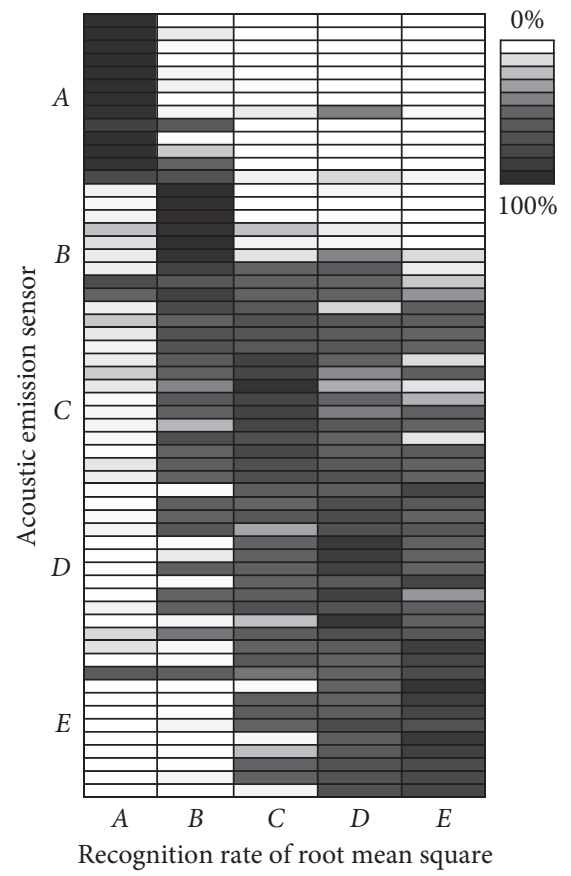

(b)

Figure 5: Color scale diagram of root mean square recognition rate.

At the same time, in Figure 5, we can see that the accuracy rate of the two adjacent stages is lower than that of the middle part of the stage.

Taking root mean square value as an example, it can be seen from Figures 3-5 that the fluctuation of life curve collected by acoustic emission sensor in the first stage is smaller than that of vibration sensor. At this time, we can see that the accuracy rate of acoustic emission sensor in the first stage is high. Compared with other stages, it can be found that the smaller the fluctuation of bearing life curve is, the higher the accuracy of bearing life stage is. On the whole, the fluctuation of bearing life curve collected by vibration sensor is smaller than that of acoustic emission sensor, so vibration sensor is better than acoustic emission sensor in bearing life prediction.

\section{Conclusion}

Aiming at the problem that a single sensor cannot reflect the bearing life information comprehensively, a bearing residual life prediction method based on multi-information fusion and convolutional neural network is proposed.

(1) This paper studies the prediction of the remaining life of subway traction motor bearings with deep learning and information fusion technology. First, use vibration sensor and acoustic emission sensor to collect bearing life signals on the bearing fatigue test bench; secondly, use wavelet packet decomposition to denoise the collected original signals and extract multiple eigenvalues. On this basis, multiple eigenvalues are extracted. The eigenvalues are normalized and the bearing degradation trend is analyzed. It is found that the vibration sensor performs better than the acoustic emission sensor in terms of dimension, and the acoustic emission sensor performs better than the vibration sensor in the dimensionless aspect. Finally, the processed multiple eigenvalues are fused and input into the convolutional neural network for training to predict the remaining service life of the bearing.

(2) The results show the following: (1) The vibration sensor has less fluctuation on the bearing life curve compared to the acoustic emission sensor, and the recognition rate of using two types of sensors at the same time is higher than that of using a single type of sensor. (2) The information fusion of different types of sensors is realized by using feature layer fusion, and the combination of convolutional neural network can effectively predict the remaining life of the bearing.

\section{Data Availability}

The data used to support the findings of this study are included within the article.

\section{Conflicts of Interest}

The authors declare that they have no conflicts of interest.

\section{References}

[1] Y. Lei, J. Lin, and M. J. Zuo, "Condition monitoring and fault diagnosis of planetary gearboxes: a review," Measurement, vol. 48, no. 1, pp. 292-305, 2014. 
[2] A. Ghods and H. H. Lee, "Probabilistic frequency-domain discrete wavelet transform for better detection of bearing faults in induction motors," Neurocomputing, vol. 188, pp. 206-216, 2016.

[3] J. Liu, W. Wang, and F. Golnaraghi, "An enhanced diagnostic scheme for bearing condition monitoring," IEEE Transactions on Instrumentation and Measurement, vol. 59, pp. 309-321, 2010.

[4] Y. Wang, G. Xu, Q. Zhang, D. Liu, and K. Jianga, "Rotating speed isolation and its application to rolling element bearing fault diagnosis under large speed variation conditions," Journal of Sound and Vibration, vol. 348, pp. 381-396, 2015.

[5] F. Jia, Y. Lei, J. Lin, X. Zhou, and N. Lu, "Deep neural networks: a promising tool for fault characteristic mining and intelligent diagnosis of rotating machinery with massive data," Mechanical Systems and Signal Processing, vol. 72, pp. 303315, 2016.

[6] W. Deng, J. Xu, X.-Z. Gao, and H. Zhao, "An enhanced MSIQDE algorithm with novel multiple strategies for global optimization problems," IEEE Transactions on Systems, Man, and Cybernetics: Systems, pp. 1-10, 2020.

[7] X. Cai, H. Zhao, S. Shang et al., "An improved quantuminspired cooperative co-evolution algorithm with mulistrategy and its application," Expert Systems with Applications, vol. 171, Article ID 114629, 2021.

[8] W. Deng, S. Shang, X. Cai et al., "Quantum differential evolution with cooperative coevolution framework and hybrid mutation strategy for large scale optimization," KnowledgeBased Systems, vol. 224, Article ID 107080, 2021.

[9] J. Wen, H. Gao, and J. Zhang, "Bearing remaining useful life prediction based on a nonlinear wiener process Model," Shock and Vibration, vol. 2018, Article ID 4068431, 13 pages, 2018.

[10] X. Chen, Z. Shen, Z. He et al., "Remaining life prognostics of rolling bearing based on relative features and multivariable support vector machine," in Proceedings of the Institution of Mechanical Engineers-Part C: Journal of Mechanical Engineering Science, vol. 227, pp. 2849-2860, January 2013.

[11] H. Zhao, H. Liu, Y. Jin, X. Dang, and W. Deng, "Feature extraction for data-driven remaining useful life prediction of rolling bearings," IEEE Transactions on Instrumentation and Measurement, vol. 70, pp. 1-10, 2021.

[12] W Yang and R Court, "Experimental study on the optimum time for conducting bearing maintenance," Measurement, vol. 46, no. 8, pp. 2781-2791, 2013.

[13] Q. Wu and C. Zhang, "Cascade fusion convolutional longshort time memory network for remaining useful life prediction of rolling bearing," IEEE Access, vol. 8, pp. 3295732965, 2020.

[14] F. Wang, X. Liu, C. Liu, H. Li, and Q. Han, "Remaining useful life prediction method of rolling bearings based on pchipEEMD-GM(1, 1) model," Shock and Vibration, vol. 2018, Article ID 3013684, 10 pages, 2018.

[15] Y. Cekic, "Bearing fault detection by four-band wavelet packet decomposition," Technology News Focus, vol. 23, no. 1, pp. 91-98, 2019.

[16] S. Fei, "Fault diagnosis of bearing based on wavelet packet transform-phase space reconstruction-singular value decomposition and SVM classifier," Arabian Journal for Science and Engineering, vol. 42, pp. 1967-1975, 2017.

[17] L. Ren, Y. Sun, J. Cui, and L. Zhang, "Bearing remaining useful life prediction based on deep autoencoder and deep neural networks," Journal of Manufacturing Systems, vol. 48, pp. 71-77, 2018.
[18] X. Pang, D. Wang, L. Chen, and L. Ye, "Grey relation evaluation and truth value fusion of rolling bearing multisource residual stress," Mathematical Problems in Engineering, vol. 2020, Article ID 3401385, 15 pages, 2020.

[19] Y. Xu, W. Cai, and T. Xie, "Fault diagnosis of subway traction motor bearing based on information fusion under variable working conditions," Shock and Vibration, vol. 2021, Article ID 5522887, 12 pages, 2021. 